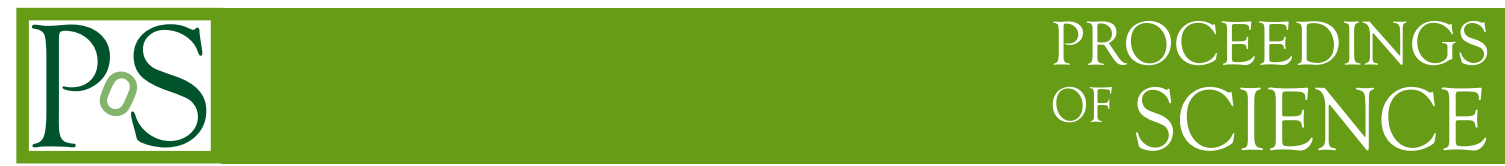

\title{
Phase structure of strongly interacting matter
}

\author{
Stefan SCHRAMM* \\ FIAS, Goethe-University Frankfurt \\ E-mail: schrammeth.physik.uni-frankfurt.de \\ Jan Steinheimer-Froschauer \\ Institut für Theoretische Physik, Goethe-University Frankfurt \\ E-mail: steinheimer@th.physik.uni-frankfurt.de
}

\begin{abstract}
We discuss an effective chiral SU(3) model that includes hadronic as well as quark degrees of freedom. Whereas the expectation values of the scalar mesonic fields correspond to the order parameter of chiral symmetry restoration a field representing the Polyakov loop describes the deconfinement process. Excluded volume effects for the hadrons are taken into account. We present numerical results for zero chemical potential. Comparisons to lattice results are shown.
\end{abstract}

XLVIII International Winter Meeting on Nuclear Physics in Memoriam of Ileana Iori 25-29 January 2010

Bormio, Italy

\footnotetext{
*Speaker.
} 


\section{Hadronic Model}

There are a number of effective hadronic flavor-SU(3) models that describe matter properties around the nuclear ground state quite well (e.g. [1] and references therein). Various extended quark model approaches have been developed in the recent years for the study of the high temperature or density regime and the deconfinement phase transition [2,3]. To investigate the chiral and deconfinement phase transitions, where hadrons and quarks are involved, and in order to have a coherent equation of state that covers the whole range of chemical potentials and temperatures relevant for ultrarelativistic heavy-ion collisions a combined hadron-quark model can serve as a very useful approach to this problem. In the following we outline such a hadron-quark formalism.

To ensure that the model has a reasonable behavior at low densities and low temperatures we adopt a well-tested extended flavor $\mathrm{SU}(3) \sigma-\omega$ model as basis for the description of the hadronic system. As hadronic degrees of freedom we include the lowest baryonic and mesonic $\mathrm{SU}(3)$ multiplets.

The Lagrange density of our extended non-linear $\sigma-\omega$ model reads:

$$
L=L_{\text {Kin }}+L_{\text {Int }}+L_{S e l f}+L_{S B},
$$

with the kinetic energy term $L_{K i n} . L_{S e l f}$ and $L_{S B}$ (assuming, for simplicity, isospin-symmetric matter) are given by

$$
\begin{gathered}
L_{\text {Self }}=-\frac{1}{2}\left(m_{\omega}^{2} \omega^{2}++m_{\phi}^{2} \phi^{2}\right)-g_{4}\left(\omega^{4}+\frac{\phi^{4}}{4}+3 \omega^{2} \phi^{2}+\frac{4 \omega^{3} \phi}{\sqrt{2}}+\frac{2 \omega \phi^{3}}{\sqrt{2}}\right) \\
+\frac{1}{2} k_{0}\left(\sigma^{2}+\zeta^{2}\right)-k_{1}\left(\sigma^{2}+\zeta^{2}\right)^{2}-k_{2}\left(\frac{\sigma^{4}}{2}+\zeta^{4}\right)-k_{3} \sigma^{2} \zeta-k_{4} \ln \frac{\sigma^{2} \zeta}{\sigma_{0}^{2} \zeta_{0}} \\
L_{S B}=-m_{\pi}^{2} f_{\pi} \sigma-\left(\sqrt{2} m_{k}^{2} f_{k}-\frac{1}{\sqrt{2}} m_{\pi}^{2} f_{\pi}\right) \zeta
\end{gathered}
$$

where $\omega$ and $\phi$ are the zeroth components of the isoscalar non-strange and strange vector fields, respectively. $\sigma$ and $\zeta$ are the corresponding scalar fields. $L_{\text {Self }}$ includes mass terms and quartic self-interactions of the vector mesons, as well as the self-interactions of the scalar mesons that induce the spontaneous breaking of chiral symmetry. The explicit chiral-symmetry breaking is implemented through the term $L_{S B}$ (for extended details on the full Lagrangian see [1]).

The interactions between baryons and the scalar and vector mesons are linear and read

$$
\mathscr{L}_{\mathrm{BM}}=-\sum_{i} \bar{\psi}_{i}\left(g_{i \sigma} \sigma+g_{i \zeta} \zeta\right) \psi_{i}, \quad \mathscr{L}_{\mathrm{BV}}=-\sum_{i} \bar{\psi}_{i}\left(g_{i \omega} \gamma_{0} \omega+g_{i \phi} \gamma_{0} \phi\right) \psi_{i}
$$

The index $i$ sums over the baryon octet. Vacuum and in-medium masses of the baryons are generated via their couplings to the scalar condensates.

For the baryon-vector couplings $g_{i \omega}$ and $g_{i \phi}$ pure $f$-type coupling is assumed as discussed in [1], $g_{i \omega}=\left(n_{q}^{i}-n_{\bar{q}}^{i}\right) g_{8}^{V}, g_{i \phi}=-\left(n_{s}^{i}-n_{\bar{s}}^{i}\right) \sqrt{2} g_{8}^{V}$, where $g_{8}^{V}$ denotes the vector coupling of the baryon octet and $n^{i}$ the number of constituent quarks of species $i$ in a given hadron. The relative couplings are in accordance with additive quark model constraints. 
The parameters of the purely hadronic model are fixed by symmetry relations, hadronic vacuum observables and nuclear matter saturation properties (see [1]). In addition, the model also provides a satisfactory description of realistic finite-size and/or isospin asymmetric systems like nuclei and neutron stars $[4,5]$.

\section{Implementing Quarks}

For a realistic description of the system at high temperatures and densities we introduce quark fields into our model following the general approach outlined in $[2,3]$.

The quarks couple to the mean fields of the model. As order parameter for the deconfinement transition we introduce an effective Polyakov loop field $\Phi$ and its conjugate $\Phi^{*}$ with a potential for the field reading [3]:

$$
U=-\frac{1}{2} a(T) \Phi \Phi^{*}+b(T) \ln \left[1-6 \Phi \Phi^{*}+4\left(\Phi^{3} \Phi^{* 3}\right)-3\left(\Phi \Phi^{*}\right)^{2}\right]
$$

where $a(T)=a_{0} T^{4}+a_{1} T_{0} T^{3}+a_{2} T_{0}^{2} T^{2}, b(T)=b_{3} T_{0}^{3} T$. The parameters are fitted to lattice QCD results at zero chemical potential.

The Polyakov loop couples to the quarks via their partition functions:

$$
\Omega_{q}=-T \sum_{i \in Q} \frac{\gamma_{i}}{(2 \pi)^{3}} \int d^{3} k \ln \left(1+\Phi \exp \frac{E_{i}^{*}-\mu_{i}}{T}\right)
$$

and

$$
\Omega_{\bar{q}}=-T \sum_{i \in Q} \frac{\gamma_{i}}{(2 \pi)^{3}} \int d^{3} k \ln \left(1+\Phi^{*} \exp \frac{E_{i}^{*}+\mu_{i}}{T}\right)
$$

Quark contributions in the confined phase with $\Phi=0$ are thus suppressed. In order to ensure that hadronic degrees of freedom vanish at high temperatures we include a finite volume correction $v$ for the hadrons $\left(v=1 \mathrm{fm}^{3}\right)$ extending the thermodynamically consistent approach discussed in e.g. [6]. Thus we get

$$
v_{\text {Quark }}=0, v_{\text {Baryon }}=v, v_{\text {Meson }}=v / 8
$$

assuming, for simplicity, a meson radius of half the size of the effective baryon radius. At this stage we do not include more subtle effects in the description of the excluded volume effects like possible Lorentz contractions or temperature-dependent effects [7]. An alternative approach to suppress hadrons at high temperatures and densities by generating a mass shift for the hadronic particles at non-zero values of $\Phi$ is discussed in ref. [8].

Using a modified chemical potential $\widetilde{\mu}_{i}$ for particle $i, \widetilde{\mu}_{i}=\mu_{i}-v_{i} P$, where $P$ is the total pressure, and by correcting all energy, particle, and entropy densities $\left(\widetilde{e_{i}}, \widetilde{\rho_{i}}\right.$ and $\left.\widetilde{s_{i}}\right)$ by the volume exclusion factor:

$$
f=\frac{V^{\prime}}{V}=\left(1+\sum_{i} v_{i} \rho_{i}\right)^{-1}
$$

one obtains a consistent set of equations. 


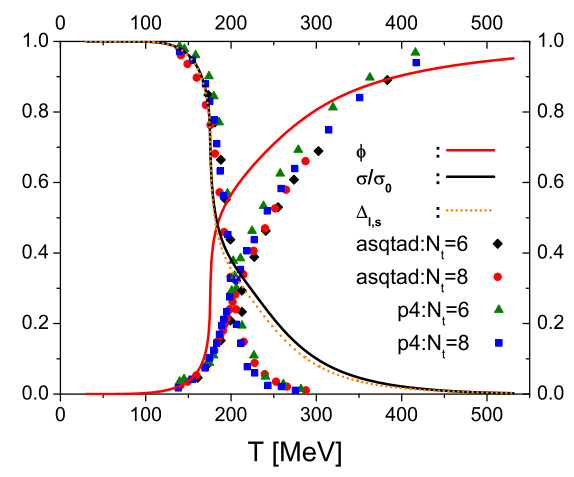

Figure 1: Scalar condensate and Polyakov loop as function of temperature compared to lattice results [10].

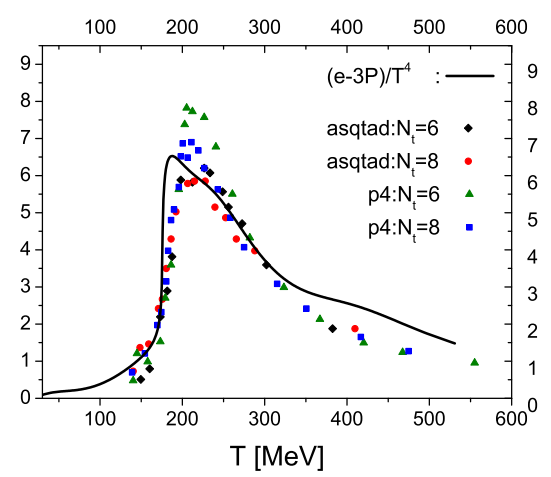

Figure 2: Interaction measure as function of temperature compared to lattice results using different time slices and effective actions[10].

\section{Results}

Solving for the fields by extremizing the grand canonical potential in mean-field approximation including thermal mesons we determine particle densities and thermodynamical quantities as function of temperature and chemical potential [9]. The plots show results for vanishing chemical potential. Finite-density results will be presented in forthcoming publications. Fig. 1 shows the temperature dependence of the scalar condensate and the Polyakov loop, representing the chiral restoration and deconfinement transitions. The critical temperature in the model is $T_{c}=175 \mathrm{MeV}$, defined as maximum in the change of the scalar condensate during the cross-over phase transition. The curves are in qualitative agreement with lattice gauge results also shown in the plot [10] Through the effective potential for the Polyakov loop also the gluonic contributions to energy density and pressure are included. In Fig. 2 the so-called interaction measure is plotted, defined as deviation of the thermodynamical quantities from an ideal gas behavior $(\varepsilon-3 p) / T^{4}$. The model shows the same behavior as the lattice results, with a peak in the interaction measure closely above $T_{c}$. The resulting hadronic and quark densities are plotted in Fig. 3. Around the "critical" $T_{c}$ a mixture of hadrons and quarks can be observed, which is quite realistic given the smooth behavior 


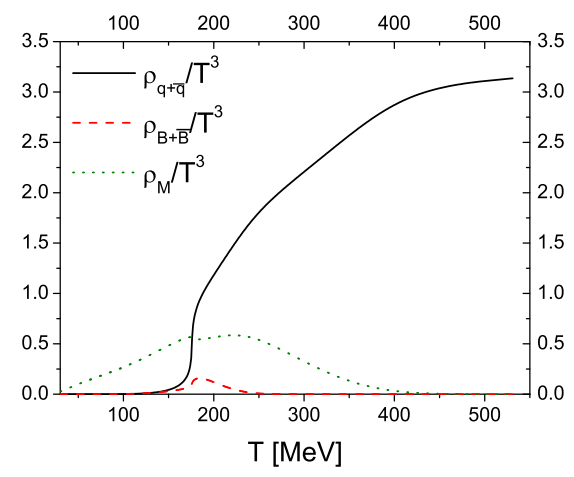

Figure 3: Particle number densities over $T^{3}$ for different particle species as function of $T$. The solid line shows the total number density of quarks and antiquarks. The dotted line refers to the total meson density and the dashed line to the number density of baryons and antibaryons.

of the phase transition and the still quite low value of the Polyakov loop field in this regime as also observed on the lattice. At higher temperatures the system is dominated by quarks very fast.

We have developed a unified hadron-quark model and studied its thermodynamic properties at zero density. The comparison with lattice results shows reasonable agreement. Work on the model calculations for finite chemical potential and the implementation of the equation of state in hydrodynamic simulations of heavy-ion collisions are in progress.

\section{References}

[1] P. Papazoglou, D. Zschiesche, S. Schramm, J. Schaffner-Bielich, H. Stöcker and W. Greiner, Phys. Rev. C 59, 411 (1999).

[2] K. Fukushima, Phys. Lett. B 591, 277 (2004)

[3] C. Ratti, M. A. Thaler and W. Weise, Phys. Rev. D 73, 014019 (2006)

[4] S. Schramm, Phys. Rev. C66 064310 (2002).

[5] V. Dexheimer and S. Schramm, Astrophys. J. 683, 943 (2008).

[6] D. H. Rischke, M. I. Gorenstein, H. Stoecker and W. Greiner, Z. Phys. C 51, 485 (1991).

[7] J. I. Kapusta and K. A. Olive, Nucl. Phys. A 408, 478 (1983); K. A. Bugaev, Nucl. Phys. A 807, 251 (2008).

[8] V. Dexheimer and S. Schramm, NP A827, 988; V. Dexheimer and S. Schramm, submitted to PRC [arxiv:astro-ph/0901.1748]

[9] J. Steinheimer, S. Schramm, and H. Stöcker, arxiv:hep-ph/0909.4421, submitted to Phys. Rev. C.

[10] A. Bazavov et al., Phys. Rev. D 80, 014504 (2009) 